\title{
Fracture rates calculated from fracture histories in normal postmenopausal women
}

\author{
David B Cleghorn, Karen J Polley, B E Christopher Nordin
}

\begin{abstract}
Study objective-The aim was to estimate fracture rates and fracture prevalence from fracture histories in normal postmenopausal women.

Design-Apparently healthy postmenopausal women were recruited by advertising in the media. Fracture histories were obtained by personal interview in 1983 and again by interview or questionnaire in 1988. Fracture rates were calculated prospectively and retrospectively.

Participants-492 women (mean age 58.6 years) were selected from over 1000 applicants on the basis that they were suffering from no disease nor taking any therapy which might affect their bones.
\end{abstract}

Measurements and main resultsRetrospective premenopausal and postmenopausal fracture rates were calculated in 1983, prospective rates calculated from 1983 to 1988 , and retrospective rates checked again on the second occasion. The retrospective and prospective fracture rates were very similar. The five year fracture rates were low and steady until the menopause, when they rose by a factor of 10 and reached a new plateau after about 15 years. The results were comparable to those obtained from hospital statistics.

Conclusions-The menopausal rise in fracture rates not only involves wrist fractures but most peripheral fractures, and probably reflects postmenopausal loss of trabecular bone. Meaningful fracture rates can be calculated from individual fracture histories in a well defined population. This technique may be particularly useful in developing countries where public health data may be incomplete.

\footnotetext{
Department of Nutrition and Dietetics, Royal Adelaide Hospital, Adelaide, South Australia

D B Cleghorn K J Polley Institute of Medical and Veterinary Science, Frome Road, Adelaide 5000, South Australia

B E C Nordin

Correspondence to: Professor Nordin

Accepted for publication April 1991
} only ones that can be related to a deficiency of bone. irtually all studies of fracture epidemiology are developing countries, even hip froure rates uncertain because so many of these cases never reach hospital. Moreover, the published studies of the relation between osteoporosis and fracture concentrate almost exclusively on fractures of the
We have calculated the rates of peripheral non-hip fractures, retrospectively and prospectively, in a cohort of 492 apparently healthy postmenopausal volunteers from fracture histories obtained at the beginning and end of a five year period. The results indicate that the postmenopausal surge in fracture rates applies to most if not all peripheral fractures and that wrist fractures represent only about $30^{\circ}{ }_{0}$ of the total. The results suggest that fracture histories are extremely reproducible within individuals and reveal that the total fracture prevalence attributable to menopausal bone loss is substantially greater than has been implied by previous studies.

\section{Methods}

The series comprises 492 apparently normal, healthy postmenopausal volunteers with a mean age in 1988 of 63.6 (SD 5.7) years. They comprise 492 of our original cohort of 557 healthy volunteers studied in $1983^{12}$ who responded to a follow up inquiry five years later. This cohort was originally recruited through the media. They represent the residue of over 1000 applicants after exclusion by questionnaire of all those who were receiving therapies (such as diuretics or oestrogen) or were suffering from diseases (such as arthritis) which might affect bone. At a later stage, after biochemical tests on blood and urine, cases of hyperparathyroidism, Paget's disease, and other disorders were excluded-as were subjects who were found to be premenopausal. The final entry into the study then comprised 557 women. The present 492 represent all those who responded to follow up enquiries in 1988. Three hundred and seventy one had undergone a natural menopause; 63 had been oophorectomised and 58 had undergone simple hysterectomy. In the 434 in whom the age at menopause could be defined, the mean years since menopause in 1988 was 14.4 (SD 6.5 ) in the whole set, 13.9 (SD 6.4) in the natural menopause set, and 18.6 (SD 7.0) in the oophorectomised set. The mean age at menopause was $50 \cdot 1$ (SD 4.3) years in the natural menopause set and 43.9 (SD 6.2) in the oophorectomised set.

Fracture histories were ascertained by self report. In 1983, the volunteers were questioned about fractures they had incurred since the age of 18 years and asked to recall the age at fracture and the type of fracture. Fractures associated with road traffic accidents were excluded. In 1988, the same information was obtained again from 360 who attended for personal interview and 134 who replied to a questionnaire, but two of the latter were excluded because they could not recall their age at fracture. The 1988 survey therefore

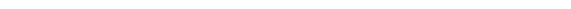


provided a check on the original information as well as new information about fresh fractures since 1983. There were only four early fractures reported in 1988 which had not been reported in 1983. The reproducibility of the fracture histories after a five year interval was therefore extremely high.

Age specific fracture rates were calculated by using as the population at risk all those subjects over the specified age at the time of interview. Thus the fracture rate at age 60 years was calculated from the number of fractures reported to have occurred at that age divided by the number of women who were more than 60 years old at the time of interview.

\section{Results}

The total number and types of fracture which occurred before 1983 and in the five years 19841988 are shown in table I. There had been 45 fractures in 39 subjects before the menopause; 114 in 84 subjects after the menopause up to 1983; and 69 in 57 subjects in the next five years. Ninety six had one fracture, 36 had two fractures, 12 had three fractures, one had four fractures, and four had five fractures, making a total of 228 fractures in 149 subjects altogether. The mean definable age at menopause of the fracture subjects was $49 \cdot 2$ years and of the non-fracture subjects 48.4 years (NS).

Before the menopause, the most common single fracture was of the foot or ankle (table I). After the menopause, in both retrospective and prospective studies, the commonest single fracture was the wrist fracture, accounting for about $30 \%$ of the total (table I). This was followed by ankle (foot) fractures and rib fractures. There was only one hip fracture in the whole series.

Of the 39 women with fractures before the menopause, $13(33 \%)$ had at least one subsequent fracture after the menopause. Of the 453 who did not fracture before the menopause, $110(24.3 \%)$ fractured subsequently (NS).

Of the 159 fractures which occurred up to 1983 , 114 occurred in 84 women after the menopause.

Table I Fracture type and numbers in 492 subjects

\begin{tabular}{lccc}
\hline & Premenopause & $\begin{array}{l}\text { Postmenopause } \\
\text { up to } 1983\end{array}$ & $\begin{array}{c}\text { Postmenopause } \\
1984 \text { to } 1988\end{array}$ \\
\hline Wrist & $7(16 \%)$ & $37(32 \%)$ & $19(28 \%)$ \\
Arm & $1(2 \% \%)$ & $7(6 \%)$ & $9(13 \% \%)$ \\
Hand, finger & $1(2 \%)$ & $7(6 \%)$ & $4(6 \% \%)$ \\
Rib & $6(14 \%)$ & $17(15 \%)$ & $9(13 \% \%)$ \\
Ankle, foot, toe & $20(44 \%)$ & $26(23 \% \%)$ & $20(29 \%)$ \\
Other & $10(22 \%)$ & $20(18 \%)$ & $8(11 \% \%)$ \\
Fracture subjects & 39 & 84 & 57 \\
Total fractures & 45 in about 30 years & 114 in 9.4 years & 69 in 5 years \\
Fracture rate per & & & \\
$\quad$ & 374 & 2465 & 2805 \\
\hline
\end{tabular}

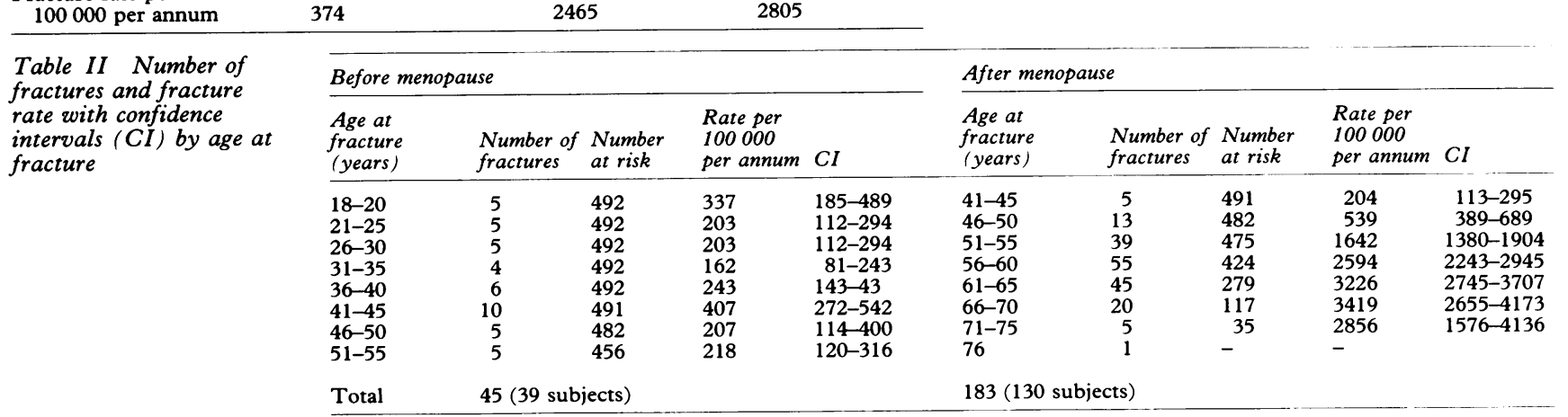

Since the mean years since menopause in 1983 was $9 \cdot 4$, the postmenopausal fracture rate was $114 / 492$ in 9.4 years or 2465 per 100000 per annum (table I).

In the next five years, there were 69 further fractures (in 57 women), making a fracture rate of $69 / 492$ in five years or 2805 per 100000 per annum (table I). Of the 84 subjects who experienced postmenopausal fractures before $1984,18(21 \%)$ had one or more further fractures in the next five years; of the 408 who did not experience postmenopausal fractures before 1984, $39\left(10^{\circ}\right)$ had one or more fractures subsequently. This difference is significant $(p<0.005)$.

The age specific fracture rates in the whole set are shown in table II and in the figure. The numerators are the number of fractures grouped by age at fracture and the denominators the number of women in the study who were at risk at that time, both scaled up to rates per 100000 per annum. The rates are steady at about 200 to 300 per 100000 per annum before the menopause, climb after the menopause to about 3000 per 10000 per annum, and then plateau at about age 60 years (figure).

\section{Discussion}

The great majority of women appear to remember their fractures and the age at which they occurred with remarkable clarity. Only two of our subjects felt unable to define the age at which fracture occurred (and were therefore excluded) and only four gave significantly different pre-1983 fracture histories in 1983 and 1988.

The retrospective and prospective parts of our study yielded very similar fracture rates, although the subjects were of course five yers older in the second study. Since the fracture rate was so stable and there were 57 women out of 492 who fractured in the five years 1984-1988, we infer that at least $50 \%$ of women suffer a non-hip peripheral fracture in the 25 years following the menopause. Many of these have more than one fracture since a postmenopausal fracture increased the risk of subsequent fracture by a factor of two and this was very significant.

The fracture rates we have calculated from fracture histories confirm but extend the fracture rates calculated in other ways. Thus previous workers ${ }^{3-5}$ have reported wrist fractures in young adult women of about 50 to 75 per 100000 per annum, which is compatible with our total premenopausal fracture rate of about 250 per 100000 per annum since wrist fractures comprise some $16 \%$ of all premenopausal fractures. Previous workers have also shown a steep rise in 


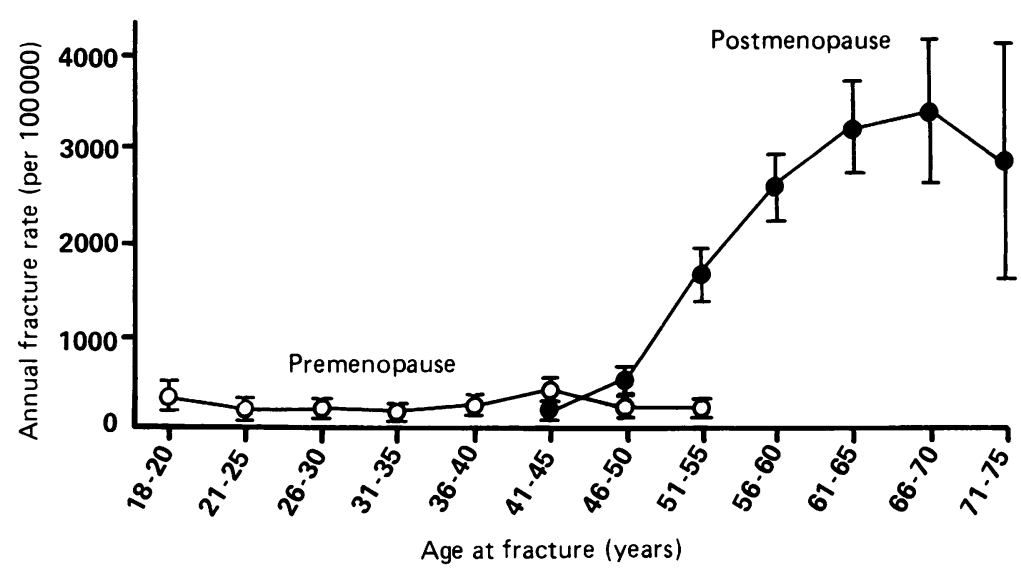

Annual fracture rates by five year age groups calculated from fracture histories in 492 healthy postmenopausal volunteers

wrist fracture rates to about 700 per 100000 per annum in women over age 50 years, followed by a plateau. Our peripheral fracture rates rise steeply at the menopause and plateau at age 60 years at about 3000 per 100000 per annum or about four times the published wrist fracture rate, which is compatible with our observation that wrist fractures constitute about $30^{\circ}{ }_{0}$ of postmenopausal fractures. Thus the plateau we see in our data, which comprise all peripheral non-hip fractures, is comparable to that observed in other studies based on the wrist fracture. It therefore appears that other fractures besides those at the wrist are significantly related to the menopause, presumably because the loss of trabecular bone which starts at that time is not confined to the forearm. These other fractures (which include only one hip fracture because of the relative youth of our subjects) probably follow the epidemiological pattern of the wrist fracture, since the proportion of each fracture type is much the same in the retrospective and prospective studies (table I). However, the small number of some fractures and the age limit in our series preclude any definitive statement on this issue.

The main risk factor for all these fractures is almost certainly the loss of bone after the menopause; we have reported elsewhere the significant bone deficit in the fracture cases of this series. ${ }^{6}$ Menopausal loss of bone (probably mainly trabecular) is exponential in type and self limiting. ${ }^{7}$ It is this self limiting feature (which has been noted by others ${ }^{8-10}$ ) which accounts for the plateau in peripheral fracture rates from about age 60 years onwards. Peripheral fractures in this context do not include hip fractures, which display a very different pattern, namely a logarithmic rise from about age 70 years onwards. ${ }^{3}$ The rise in hip fracture rates in old women probably reflects the linear loss of cortical bone with age which starts at about 55 years ${ }^{7}$ and is superimposed on the earlier trabecular bone loss. In other words, trabecular bone loss alone increases the risk of non-hip peripheral fracture but cortical bone loss is required in addition before the hip fracture rates rise.

Thus the difference in the age pattern of different fracture rates, already noted long ago between wrist and hip fractures, ${ }^{3}$ is perhaps a difference between the pattern of hip fractures and most of the other peripheral fractures. If so, osteoporotic fractures can be classified into those of the spine, non-hip periphery, and hip. The spinal and peripheral non-hip fractures represent Riggs' type I osteoporosis and the hip fractures his type II, ${ }^{11}$ but the two types probably represent simply different stages in bone loss with different emphases on trabecular and cortical bone.

Our data shed no light on vertebral fracture incidence since the subjects did not undergo spinal radiography, but cases of known or suspected spinal osteoporosis were removed from the study at an early stage, and the original cohort of 557 women (from which the present 492 were drawn) did not, to our knowledge, contain any such cases. By 1988, there were six women who reported a vertebral fracture but in the absence of radiographs of the whole set, this information is of little value and vertebral fractures were therefore not included in the present analysis.

We conclude that self reporting is an inexpensive, valuable tool in the study of fracture rates which may be particularly applicable to countries where biomedical statistical data are inadequate.

1 Nordin BEC, Polley KJ. Metabolic conseqences of the menopause. Calcif Tissue Int 1987; 41: S1-60.

2 Nordin BEC, Chatterton BE, Walker CJ, Wishart J. The relation of forearm mineral density to peripheral fractures in postmenopausal women. Med f A Aust 1987; 146: 300-4.

3 Knowelden J, Buhr AJ, Dunbar O. Incidence of fractures in persons over 35 years of age. A report to the MRC Working 18: $130-41$.

4 Boyce WJ, Vessey MP. Rising incidence of fracture of the

5 Mroximal femur. Lancet 1985; i: $150-1$. Melton LJ, Riggs BL. Epidemiology of age-related
fractures. In: Avioli LV, ed. The Osteoporotic syndrome: detection, prevention and treatment. New York: Grune and Stratton, 1987: 1-30.

6 Cleghorn DB, Polley KJ, Bellon MJ, Chatterton J, Baghurst PA, Nordin BEC. Fracture rates as a function of forearm mineral density in normal postmenopausal women. Calcif Tissue Int 1991; 49: 161-3.

7 Nordin BEC, Need AG, Chatterton BE, Horowitz M, Morris HA. The relative contributions of age and years since menopause to postmenopausal bone loss. 7 Clin Endocrinol Metab 1990; 70: 83-8.

8 Johnston CC, Norton JA, Khairi RA, Langcope C. Agerelated bone loss. In: Barzel US, ed. Osteoporosis II. New York: Grune and Stratton, 1979: 91-109.

9 Gallagher JC Goldgar D, Moy A. Total bone calcium in normal women: effect of age and menopause status. $\mathcal{F}$ Bone Miner Res 1987; 2: 491-6.

10 Nilas L, Christiansen C. Bone mass and its relationship to age and the menopause. $\mathcal{F}$ Clin Endocrinol Metab 1987; 65: age and the

11 Riggs BL, Melton J III. Evidence for two distinct syndromes of involutional osteoporosis. Am f Med 1983;
75: 899-901. 